\title{
References
}

[1] Russell S. J. Artificial intelligence: a modern approach / S. J. Russell - Pearson, 2010. - 1152 p.

[2] Goodfellow I., Bengio Y., Courville A. Deep Learning. MIT Press, 2016. 781 p.

[3] Haykin S. Neyronnyie seti: polnyiy kurs. Moskva: Vilyams, 2006. 1104 p.

[4] Kumar K. V. Neural networks and fuzzy logic. S. K. Kataria \& Sons, 2016. 300 p.

[5] CS231n: Convolutional Neural Networks for Visual Recognition. [Electronic resource]. - Access mode: http://cs231n.github.io

[6] Bertsekas D. P. Convex Optimization Theory. Athena Scientific, 2009. 256 p.

[7] Nielsen M. Neural Networks and Deep Learning. Determination Press, 2015. 216 p.

[8] Louizos C., Welling M., Kingma D. P. Learning sparse neural networks through $\mathrm{L}_{0}$ regularization. Internation. Conf. on Learning Representations, 2018.

[9] Srivastava N., Hinton G., Krizhevsky A., Sutskever I., Salakhutdinov R. Dropout: A Simple Way to Prevent Neural Networks from Overfitting. Journal of Machine Learning Research. 2014. 15. P. 1929-1958.

[10] Caruana R., Lawrence S., Giles L. Overfitting in Neural Nets: Backpropagation, Conjugate Gradient, and Early Stopping. Neural information processing systems conference, 2000.

[11] Credit Card Fraud Detection. [Electronic resource]. - Access mode: https://www.kaggle.com/mlg-ulb/creditcardfraud

[12] Abdi. H. \& Williams, L.J. Principal component analysis. [Electronic resource]. - Access mode: https://arxiv.org/pdf/1108.4372.pdf

[13] Keilwagen, J., Grosse, I., Grau, J. Area under precision-recall curves for weighted and unweighted data. PLoS One, 2014.

[14] Ting, Kai Ming. Encyclopedia of machine learning. Springer, 2010. 45p.

УДК 681.787.6

\section{ВОЛОКОННО-ОПТИЧЕСКАЯ СИСТЕМА КОНТРОЛЯ ТЯГИ ГАЗОТУРБИННЫХ ДВИГАТЕЛЕЙ}

\author{
Сандлер А.К. ${ }^{1}$, Карпилов А.Ю. ${ }^{2}$ \\ Национальный университет "Одесская морская академия", г. Одесса, Украина \\ E-mail: ${ }^{1}$ albertsand4@gmail.com, ${ }^{2}$ kau.onma@gmail.com
}

Copyright (C) 2018 by author and the journal “Automation technologies and business - processes. This work is licensed under the Creative Commons Attribution International License (CC BY). http://creativecommons.org/licanses/by/4.0
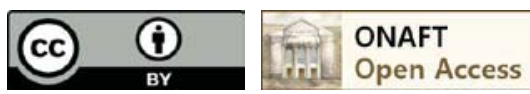

DOI:

Аннотация. На современном этапе эволючии судовой энергетики отмечается определенный рост применения газотурбинных установок (ГТУ). Анализ эксплуатации судов и кораблей с ГТУ показал, что эти установки более эффективны по сравнению с дизельныли на быстроходных (со скоростью хода более 20 ... 25 узлов) судах водоизмещуаюегго типа, а также на судах с динамическими принципами поддержания с больиой энерговооруженностью - до 20 ... 40 кBm на 1 тонну водоизмещения против 1 ... 2 кBm на 1 тонну у водоизмещающих судов со скоростями движения 12 ... 15 узлов.

Для судов и кораблей с динамическими принципами поддержания актуальным является точное определение векторов тяги двигателя. Традиционно измерение усилий, передаваемых на корпус, производится в узлах крепления установки либо на специильных динамометрических стендах.

В обоих вариантах контроля сложным является выбор тягоизмерительных устройств (ТИУ), обеспечивающих достаточно высокую информативность и чувствительность измерений. 
Анализ существующих ТИУ показал, что применение их для контроля вектора тяги ГТУ в специальных эксплуатационных условиях характеризуется недостаточной достоверностью результатов измерения.

В сложившейся ситуации представилась иелесообразной разработка нового схемотехнического решения ТИУ. Конструкция устройства позиционировалась как такая, в которой отсутствует необходимость постоянной поддержки чистоты и геометрии открытого измерительного канала, обеспечена инвариантность к помехам электромагнитного и термического происхождения, обеспечены измерения боковых составляющих тяги в двух плоскостях и одновременно сохранены надежность, чувствительность и простота схемотехнических решений систем известных типов.

Основное отличие предлагаемого устройства заключается в том, что вертикальный подвес дополнительно закреплен на горизонтальном упругом подвесе, а в тело обеих подвесов вмонтированы волоконные световоды. Световоды, играющие роль сенсорных элементов, чувствительны к деформации кручения, имеют отражающие слои на концах и термокомпенсационные биметаллические оболочки.

Комбинация оптико-механичных элементов в разработанном ТИУ позволит обеспечить:

повышенную чувствительность и точность измерения боковых составляюших вектора тяги путем исключения влияния неконтролируемых электромагнитных помех, которые создает двигатель, и силового влияния токов, генерируемых в кабелях питания;

отсутствие влияния неконтролируемых климатологических факторов на оптический канал;

зашищенность чувствительных элементов системы;

постоянность геометрии оптического канала в условиях влияния неконтролируемых эксплуатационных факторов;

постоянное измерение в реальном масштабе времени.

Использование предлагаемого ТИУ позволит адекватно и достоверно оценивать результаты испытаний газотурбинных установок.

Abstract. At the present stage of the evolution of ship energy, there is a certain increase in the use of gas turbine units (GTU). Analysis of the operation of ships and ships with gas turbine engines showed that these facilities are more efficient than diesel engines on high-speed (with a speed of more than 20 ... 25 knots) displacement-type vessels, as well as on vessels with dynamic principles of maintenance with a large power ratio - up to 20 ... $40 \mathrm{~kW}$ per 1 ton of displacement versus 1 ... 2 $k W$ per 1 ton of displacement vessels with speeds of $12 \ldots 15$ knots.

For ships and ships with dynamic maintenance principles, accurate determination of engine thrust vectors is relevant. Traditionally, the measurement of the forces transmitted to the housing is carried out in the attachment points of the installation or on special dynamometric stands.

In both control options, it is difficult to choose a load measuring device (TIU) that provides a sufficiently high information content and sensitivity of measurements.

An analysis of the existing TIUs showed that their use to control the thrust vector of a gas turbine in special operating conditions is characterized by insufficient reliability of the measurement results.

In this situation, it seemed appropriate to develop a new circuit solution TIU. The design of the device was positioned as such in which there is no need for constant support for the cleanliness and geometry of the open measuring channel, it provides invariance for interference of electromagnetic and thermal origin, measurements of lateral components of the thrust in two planes are provided, and at the same time, the reliability, sensitivity and simplicity of circuit solutions of known types of systems are preserved.

The main difference of the proposed device is that the vertical suspension is additionally mounted on a horizontal elastic suspension, and fiber optic fibers are mounted in the body of both suspensions. The optical fibers, which play the role of sensor elements, are sensitive to torsional deformation, have reflective layers at the ends and thermocompensating bimetallic shells.

The combination of optical-mechanical elements in the developed TIU will provide:

increased sensitivity and accuracy of measurement of the lateral components of the thrust vector by eliminating the influence of uncontrolled electromagnetic interference that the engine creates and the power effect of currents generated in the power cables;

lack of influence of uncontrolled climatological factors on the optical channel;

security of sensitive elements of the system;

the constancy of the geometry of the optical channel under the influence of uncontrolled operational factors;

continuous measurement in real time.

Using the proposed TIU will adequately and reliably evaluate the test results of gas turbine units.

Ключевые слова: волоконно-оптическая, измерение тяги, газотурбинный двигатель.

Key words: fiber optic, traction measurement, gas turbine engine.

\section{Введение}

На современном этапе эволюции судовой энергетики отмечается определенный рост применения газотурбинных установок (ГТУ). Анализ эксплуатации судов и кораблей с ГТУ показал, что эти установки более эффективны по сравнению с дизельными на быстроходных (со скоростью хода более 20 ... 25 узлов) судах водоизмещающего типа, а 
также на судах с динамическими принципами поддержания с большой энерговооруженностью - до 20 ... 40 кВт на 1 тонну водоизмещения против $1 \ldots 2$ кВт на 1 тонну у водоизмещающих судов со скоростями движения $12 \ldots 15$ узлов [1].

Для судов и кораблей с динамическими принципами поддержания актуальным является точное определение векторов тяги двигателя. Традиционно измерение усилий, передаваемых на корпус, производится в узлах крепления установки либо на специальных динамометрических стендах.

В обоих вариантах контроля сложным является выбор тягоизмерительных устройств (ТИУ), обеспечивающих достаточно высокую информативность и чувствительность измерений [2].

\section{Анализ литературных источников и постановка задачи}

Анализ существующих ТИУ показал, что применение их для контроля вектора тяги ГТУ в специальных эксплуатационных условиях характеризуется недостаточной достоверностью результатов измерения [3, 4].

Для поиска путей улучшения характеристик ТИУ были проанализированы конструкции наиболее распространенных устройств [3, 4].

Известная система, которая состоит из горизонтально расположенного и закрепленного на подвесе, упругого на кручение, подвижного коромысла, на одном конце которого через подвижный монтажный узел установлен испытуемый ГТУ. На другом конце коромысла размещен противовес для уравновешивания и отсчетное устройство для регистрации вращающегося момента (рис. 1) [3].

Недостатки системы, которые обусловлены применением одного подвеса и отсчетного устройства, отделенного от подвеса и коромысла:

невозможность учета составляющих вектора тяги двигателей в вертикальной плоскости;

необходимость дополнительных мер по поддержке геометрии измерительного канала;

необходимость постоянной компенсации влияния климатических и эксплуатационных факторов на достоверность работы отсчетного электромагнитного устройства.

Наиболее близкой за технической сущностью к задаче контроля вектора тяги является система, основу которой составляет горизонтально расположенное и закрепленное на упругом подвесе подвижное коромысло.

На одном конце коромысла через подвижный монтажный узел установлен испытуемый двигатель, а на другом конце коромысла размещен противовес для уравновешивания, оптическое отсчетное устройство и кронштейн. В кронштейне выполнена узкая вертикальная щель, совпадающая с осью упругого подвеса, зеркало, плоскость которого параллельная плоскости монтажного узла в месте стыка его с коромыслом (рис. 2) [4].

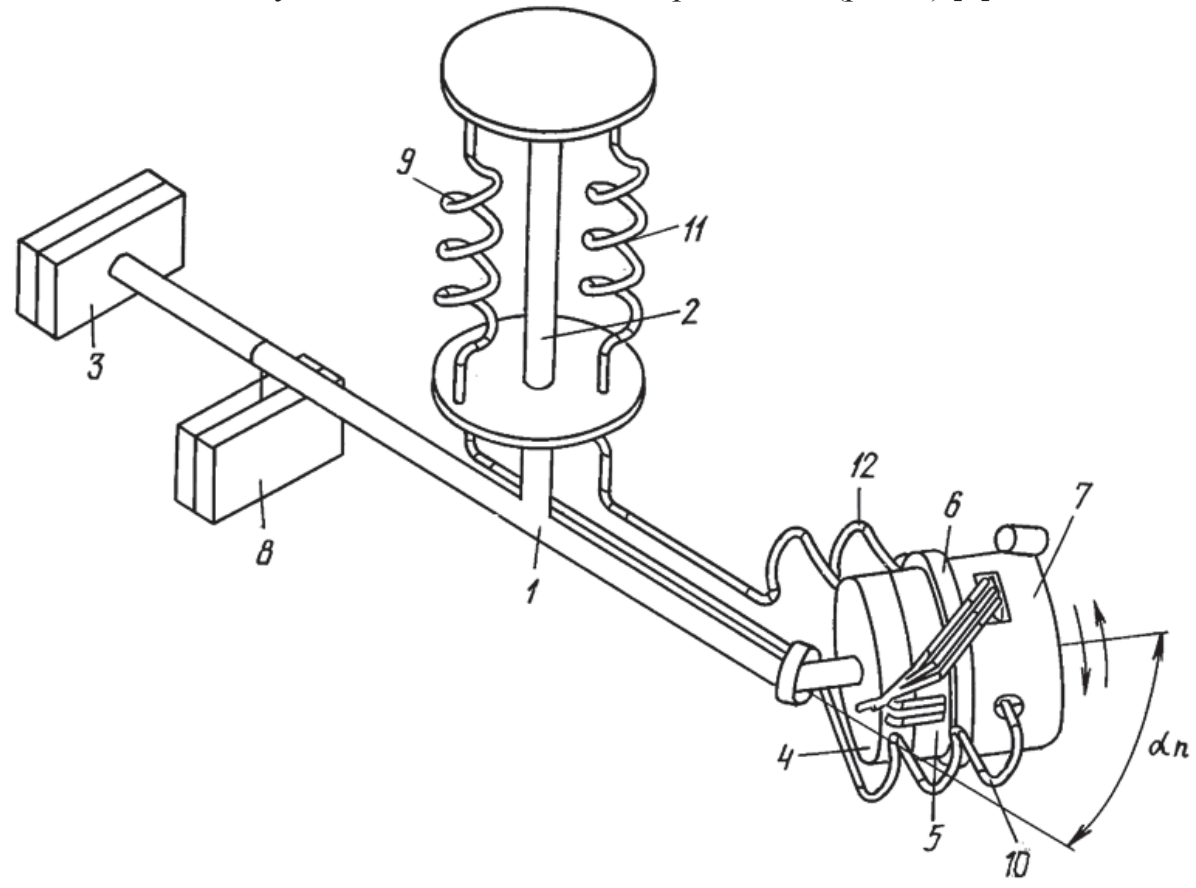

Рис. 1 - Тягоизмерительное устройство: 1 - коромысло; 2 - упругий стержень; 3 - уравновешивающая масса; 4

- привод; 5 - устройство контроля угла поворота; 6 - монтажный узел; 7 - двигатель; 8 - отсчетное устройство; 9, 10 - электрические кабели; 11, 12 - трубопроводы подачи рабочего тела 


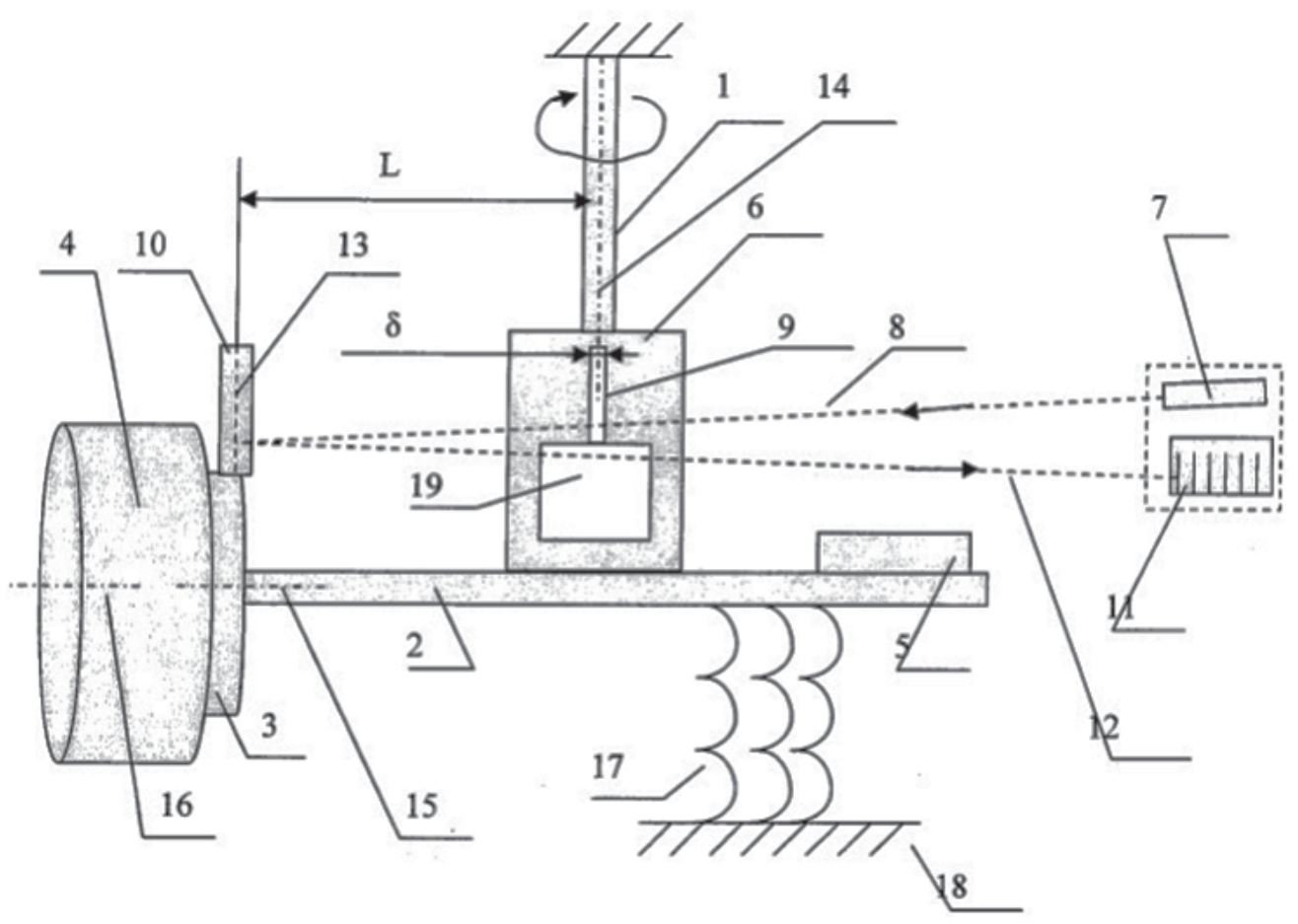

Рис. 2 - Тягоизмерительное устройство 1 - подвес; 2 - подвижное коромысло; 3 - подвижный монтажный узел;

4 - двигатель; 5 - противовес; 6 - кронштейн; 7 - источника света; 8 - луч; 9 - вертикальная щель; 10 зеркало; 11 - индикатор положения луча; 12 - отраженным от зеркала луч; 13 - вертикальный указатель; 14 - ось упругого подвеса; 15 - ось коромысла; 16 - ось двигателя

Недостатки системы, которые обусловлены применением одного подвеса, оптического отсчетного устройства, пластины со щелью и зеркала:

необходимость дополнительных мер по защите поверхности и поддержания геометрии открытого оптического измерительного канала;

невозможность учета влияния климатических и эксплуатационных факторов на результаты измерения;

невозможность учета боковых составляющих вектора тяги двигателей в вертикальной плоскости для предотвращения искажения результатов измерения.

\section{Цель и задачи исследования}

В сложившейся ситуации представилась целесообразной разработка нового схемотехнического решения ТИУ. Конструкция устройства позиционировалась как такая, в которой отсутствует необходимость постоянной поддержки чистоты и геометрии открытого измерительного канала, обеспечена инвариантность к помехам электромагнитного и термического происхождения, обеспечены измерения боковых составляющих тяги в двух плоскостях и одновременно сохранены надежность, чувствительность и простота схемотехнических решений систем известных типов.

Основное отличие предлагаемого устройства заключается в том, что вертикальный подвес дополнительно закреплен на горизонтальном упругом подвесе, а в тело обеих подвесов вмонтированы волоконные световоды. Световоды, играющие роль сенсорных элементов, чувствительны к деформации кручения, имеют отражающие слои на концах и термокомпенсационные биметаллические оболочки.

\section{Результаты исследований}

Суть предлагаемого схемотехнического решения поясняется на рис. 3.

Излучение поступает в световод, находящийся в теле подвеса. Под воздействием тяги в материале подвеса возникает динамическая деформация сдвига. Эта деформация вызывает аналогичный процесс в волоконном световоде. Вследствие кручения световода происходит изменение показателей преломления, как в сердцевины, так и оболочки световода. Происходящие изменения инициируют нарушение условий полного отражения света в световоде и создают условия для туннелирования части излучения из сердцевины в оболочку световода. Таким образом, часть света излучается за пределы световода $[6,7,8,9,10]$. 


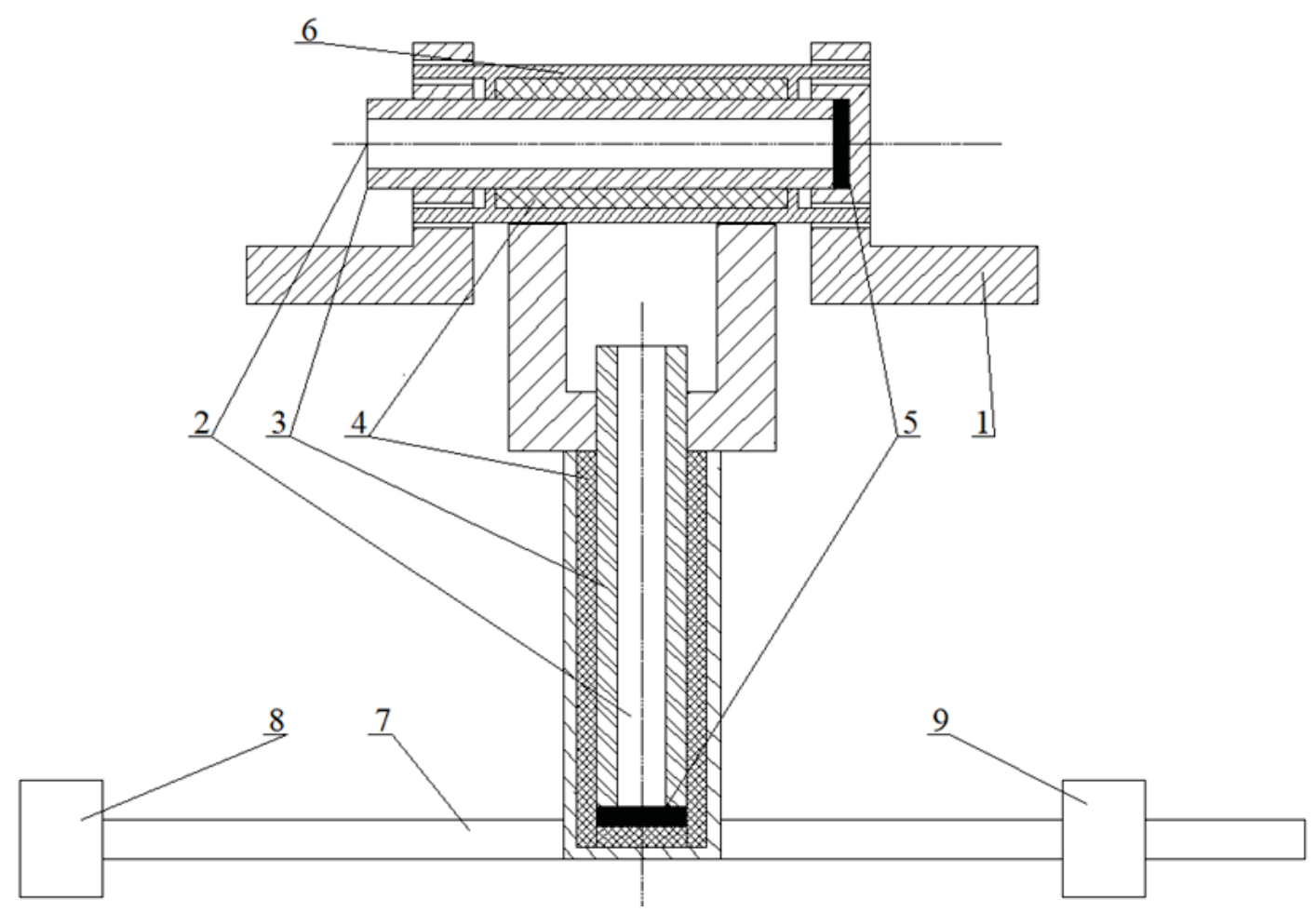

Рис. 3 - Волоконно-оптическая система контроля вектора тяги: 1 - горизонтальный подвес; 2 - волоконнооптический световод (сердцевина); 3 - волоконно-оптический световод (оболочка); 4 - термокомпенсационная биметаллическая оболочка; 5 - отражающий слой; 6 - ось горизонтального подвеса; 7 - подвижное коромысло; 8 - подвижный монтажный узел; 9 - противовес

Регистрируемая часть излучения, поступающего из световодов, расположенных в каждом из подвесов, пропорциональна величине силы тяги в соответствующей плоскости.

Для компенсации влияния температуры окружающей среды на элементы устройства применяется термокомпенсационная биметаллическая оболочка, которая пропорционально температуре, меняет изгиб световода и уменьшает заранее внесенные потери излучения в световоде.

В статическом режиме (калибрование при отсутствии тяги и уравновешенном подвижном коромысле) в блоке регистрации фиксируются соответствующие данные и поправки, учитывающие температуру окружающей среды и потери во всех элементах измерительной системы.

В динамическом режиме (измерение), после появления тяги в материале подвесов и световодов происходит динамическая деформация сдвига. Инициированное деформацией изменение показателей преломления слоев световода вызывает нарушение условий полного отражения света. Как следствие, происходит перераспределение света между направляющими и излучающими модами. Что приводит к излучению света за границы световода.

Интенсивность оставшейся части излучения будет соответствовать контролируемым изменениям вектора тяги в каждой из плоскостей контроля. А совместная обработка информации со световодов двух подвесов позволяет определить результирующий вектор тяги. Таким образом, происходит полный цикл измерения.

\section{Выводы}

Таким образом, комбинация оптико-механичных элементов в разработанном ТИУ позволит обеспечить:

повышенную чувствительность и точность измерения боковых составляющих вектора тяги путем исключения влияния неконтролируемых электромагнитных помех, которые создает двигатель, и силового влияния токов, генерируемых в кабелях питания;

отсутствие влияния неконтролируемых климатологических факторов на оптический канал;

защищенность чувствительных элементов системы;

постоянность геометрии оптического канала в условиях влияния неконтролируемых эксплуатационных факторов; постоянное измерение в реальном масштабе времени.

Использование предлагаемого ТИУ позволит адекватно и достоверно оценивать результаты испытаний газотурбинных установок. 


\section{Список использованных источников}

[1] Иванов, Р.А., Тимофеев, В.В., Шитков, В.Н. О перспективах использования газотурбинных установок на морских судах и сооружениях.// Судостроение. - 2003. - № 5. - С. 27 - 28.

[2] Кулаков, А.Д., Попов, В.В. Методы определения тяговых характеристик ГТД на летающей лаборатории и основном самолете.//Авиационно-космическая техника и технологии. - 2007. -№ 9 (45). - С. 37 - 43.

[3] Пат. 2202773 Российская Федерация, МПК7 G01L 5/00, G01M 15/00. Устройство по определению тяги и составляющих вектора тяги электрореактивного двигателя и способ испытаний./ Заявитель и патентообладатель: Федеральное государственное унитарное предприятие Российского авиационнокосмического агентства "Опытное конструкторское бюро "Факел". - № 2001100751/28; заявл.09.01.2001; опубл. 20.04.2003.

[4] Пат. 2370739 Российская Федерация, МПК7 G01L5/13 .Устройство для определения боковых составляющих вектора тяги электрореактивного двигателя. / Авторы патента: Муравлев, В. А. Салин, В. Л., Батурин, А. В., Шутов, В. Н. Патентообладатель: Федеральное государственное унитарное предприятие "Исследовательский Центр им. М.В. Келдыша". - № 2008112553/28; заявл. 03.04.2008; опубл. 20.10.2009.

[5] Бусурин, В.И., Носов, Ю.Р. Волоконно-оптические датчики: физические основы, вопросы расчета и применения. - М.: Энергоатомиздат, 1990. - 256 с.

[6] Удд, Э. Волоконно-оптические датчики. - М.: Техносфера, 2008. - 520 с.

[7] Снайдер, А., Лав, Д. Теория оптических волноводов. - М.: Радио и связь, 1987. - 656 с.

[8] Сандлер, А.К., Цюпко, Ю.М. Повнообертовий волоконно-оптичний з'єднувач. Деклараційний патент України № 110051, МПК (2016.01) G02B 6/00, G01M 11/00. - заявл. 11.03.2016. // Опубл. 26.09.2016, бюл. № 18/2016.

[9] Сандлер, А.К., Сандлер, О.А. Інваріантний волоконний акселерометр: Деклараційний патент України № 62437, МПК (2011) G01M 11/00. - заявл. 02.02.2011. // Опубл. 26.10.2011, бюл. № 18.

[10] Сандлер, А.К. Інформаційно-вимірювальні пристрої на основі волоконно-оптичних технологій. - Одесса: Издатинформ НУ "ОМА", 2018. - 165 с.

\section{References}

[1] Ivanov, R.A., Timofeev, V.V., Shitkov, V.N. (2003) O perspektivakh ispol'zovaniya gazoturbinnykh ustanovok na morskikh sudakh i sooruzheniyakh[On the prospects for the use of gas turbine units in marine vessels and structures]. Sudostroyeniye - Shipbuilding. 5. 27 - 28. [in Russian].

[2] Kulakov, A.D., Popov, V.V. (2007) Metody opredeleniya tyagovykh kharakteristik GTD na letayushchey laboratorii i osnovnom samolete [Methods for determining the thrust characteristics of a gas turbine engine in a flying laboratory and a main aircraft]. Aviatsionno-kosmicheskaya tekhnika i tekhnologii - Aerospace engineering and technology. 9 (45). 37 - 43. [in Russian].

[3] Pat. 2202773 Russian Federation, IPC7 G01L 5/00, G01M 15/00. Device for determining the thrust and components of the thrust vector of an electric jet engine and test method./ Applicant and patent holder: Federal State Unitary Enterprise of the Russian Aerospace Agency "Experimental Design Bureau" Fakel ". - No. 2001100751/28; Dec. 09.01.2001; publ. 04/20/2003.

[4] Pat. 2370739 Russian Federation, IPC7 G01L5 / 13. A device for determining the lateral components of the thrust vector of an electric jet engine. / Authors of the patent: Muravlev, V. A. Salin, V. L., Baturin, A. V., Shutov, V. N. Patent holder: Federal State Unitary Enterprise "MV Keldysh Research Center". - No. 2008112553/28; declared 04/03/2008; publ. 10/20/2009.

[5] Busurin, V.I., Nosov, YU.R.(1990). Volokonno-opticheskiye datchiki. Fizicheskiye osnovy, voprosy rascheta i primeneniya [Fiber optic sensors. Physical basis, questions of calculation and application]. Moscow: Energoatomizdat [in Russian].

[6] Udd, E. (2008) Volokonno-opticheskiye datchiki .[Fiber Optic Sensors]. Moscow: Tekhnosfera [in Russian].

[7] Snider, A., Love. J.D. (1987) Teoria opticheskih volnovodov. [Theory of optical wave guides]. Moscow: Radio i svyaz' [in Russian].

[8] Sandler, A.K., Tsyupko, Yu.M. Fully rotated fiber optic connector. Declaration Patent of Ukraine No. 110051, IPC (2016.01) G02B 6/00, G01M 11/00. - stated. 11.03.2016. // Publ. 26.09.2016, bul. № 18/2016.

[9] Sandler, A.K., Sandler, O.A. Invariant Fiber Accelerometer: Declarative Patent of Ukraine No. 62437, MPK (2011) G01M 11/00. - 02.02.2011.// Publ. 26.10.2011, bul No. 18.

[10] Sandler, A.K. (2018) Informatsiyno-vymiryuval'ni prystroyi na osnovi volokonno-optychnykh tekhnolohiy. [Information-measuring devices based on fiber-optic technologies]. Odessa: Izdatelinform NU "OMA" [in Ukraine]. 